\title{
Features of the thyroid gland morphology of some representatives of the Cervidae family, living in the Altai
}

\author{
O.E. Maltseva ${ }^{1, *}$, N.D. Ovcharenko ${ }^{2}$, N.M. Semenikhina ${ }^{3}, O . G$. Gribanova $^{4}$, and \\ I.N. Pleshakova ${ }^{5}$ \\ ${ }^{1}$ FSBEI HE Altai State Agrarian University, Barnaul, Russia, o.e.vlasova@yandex.ru \\ ${ }^{2}$ FSBEI Altai State University, Barnaul, Russia \\ ${ }^{3}$ Altai State University, Barnaul, Russia \\ ${ }^{4}$ FSBEI HE Altai State Medical University, Ministry of Health of the Russian Federation, Barnaul, \\ Russia, \\ ${ }^{5}$ FSBEI HE Altai State Agrarian University, Barnaul, Russia
}

\begin{abstract}
The authors studied the morphofunctional (anatomical and histological) features of the thyroid gland of dapple deer, maral, moose and roe deer. During the macroscopic examination of the organ, the number of lobes, the presence of an isthmus, the length, width and thickness of the lobes, and the weight of the gland were determined. During histological examination, morphometric parameters were evaluated, such as: diameter of the follicles, height of the thyrocytes, volume of the thyrocyte nuclei, and the Brown index was calculated. In all animals, the thyroid gland consisted of two lobes without an isthmus. It differs between species in the shape, size, and thickness of the lobes. The gland weight correlates with the size of the animals. Morphological differences between species consist in the heterogeneity of the location of follicles of different diameters in relation to each other. In the dapple deer, large-diameter follicles are located in the center of the organ, and smaller-diameter follicles are located both in the center and on the periphery. In roe deer, the gland follicles are almost identical in shape and size, that is, they are of the same type. The gland has a similar morphological structure in maral and moose. The colloid of the follicles has a homogeneous consistency, it is compacted and intensively stained with hematoxyl-eosin, resorption vesicles are in small quantities.
\end{abstract}

\section{Introduction}

One of ecology tasks is to study the mechanisms of adaptation to changing environmental factors. Ecological morphology considers the structure of the organs of different animal species representatives, depending on the living conditions. The characterization of the endocrine glands, which provide adaptive responses of the body is important in this aspect.

\footnotetext{
*Corresponding author: ovcharenko-55@mail.ru
} 
In addition to the theoretical value of knowing the structural differences of mammalian organs of different species, it is essential to study the structure of wild and game animals. Ungulate mammals are an important component of the Altai biocenoses. In the Altai fauna, six of the seven ungulate species that live on the territory of the Russian Federation are found in the wild: maral, reindeer, musk deer, moose, roe deer, and dapple deer [5]. The role of these species in nature is important and diverse. Being the primary consumers, they are able to exert a significant influence on the vegetation cover of the foothills and highmountain alpine meadows. Roe deer, moose, maral, and dapple deer are the main victims of secondary consumers, of which there are quite a few in these territories. In addition, marals and dapple deer are specially bred in specialized farms to produce various products.

In connection with the above, it becomes clear why the issues of biological assessment of the state of the resources of wild ungulates, the dynamics of their number, reproduction, veterinary care, the impact of human economic activity on the territorial distribution, as well as the possibility of further use of antler products of some of them are of great importance $[3,6,9]$.

In the system of the endocrine glands of the body of ungulates, one of the important places is occupied by the thyroid gland, which affects the metabolism, growth, normal physiological development of the body, reproduction $[7,10]$.

The purpose of our research was to study the morphological features of the thyroid gland among the representatives of the Cervidae family living in the Altai, in particular in the maral, roe deer, moose and dapple deer.

\section{Material and research methods}

The thyroid gland obtained from adult sexually mature males of marals, moose, roe deer and dapple deer (five animals each) for the period from December to January was used as the material for the study. After the gland was removed, weight on the scale, length, width and thickness of the lobes and the isthmus (if any) were measured using a caliper. For histological examination, pieces were cut from the middle part of the gland of the left lobe and placed in a fixing liquid - a $10 \%$ solution of formalin. The histologic diagnosis was carried out in several portions of isopropyl alcohol, followed by filling in paraffin in an automatic Medite Duo station. Paraffin sections with a thickness of 5-7 microns were stained with Ehrlich's hematoxylin - eosin.

Histological sections with a thickness of 5-7 microns were made on a rotary microtome Termo NM 525, and stained with Ehrlich's hematoxylin and 1\% alcohol-water solution of eosin. The resulting preparations were studied using a Zeiss Imager 1 microscope and a Zeiss video camera. Morphometric studies were performed in the AxioVision SE64 program. At least 60 follicles were examined in 3 fields of view from different sides of the preparation. The size (large, medium, small), length, width, and diameter of the follicles were determined. The height of epithelial cells was determined in at least 20 thyrocytes in 10 visual fields, the state of the colloid was assessed visually (color, density, presence of vacuoles), the Brown index according to the formula $\mathrm{IB}=\mathrm{Df} / 2 \mathrm{Ht}$, where $\mathrm{Df}$ is the average diameter of the thyroid follicle, microns; $\mathrm{Ht}$ is the average height of the thyrocyte, microns. The obtained data were subjected to standard statistical processing in the program Statistica CZ 7.

\section{Results and discussion}

The specific features of the thyroid gland are described in many vertebrates. The analysis of the works shows that its external structure can be fundamentally different - shape of the 
lobes, consistency, presence or absence of the isthmus. The thyroid gland of roe deer, maral, moose and dapple deer is paired, it does not have the isthmus. It is dense, compact, and has a granular structure on the cut. The gland lies dorsally on the trachea, to the side of the innominate cartilage; its caudal end reaches the third tracheal cartilage (in dapple deer, moose, roe deer and maral) and can reach 5-6 tracheal cartilage (this type of gland structure is found in maral - option 2). The weight of the thyroid gland correlates with the weight of the animal.

In the maral, the thyroid gland is red-brown, dense, and has a shiny surface. According to the shape of the lobes in different animals, there is a duality: the first option is a flattened, elongated oval shape (Figure 1). The vertical size of the lobe is $5.40 \pm 1.20 \mathrm{~cm}$, the transverse size is $2.50 \pm 0.78 \mathrm{~cm}$, the thickness is $1.3 \mathrm{~cm}$, the weight of one lobe is $7.25 \pm 1.25$ g.

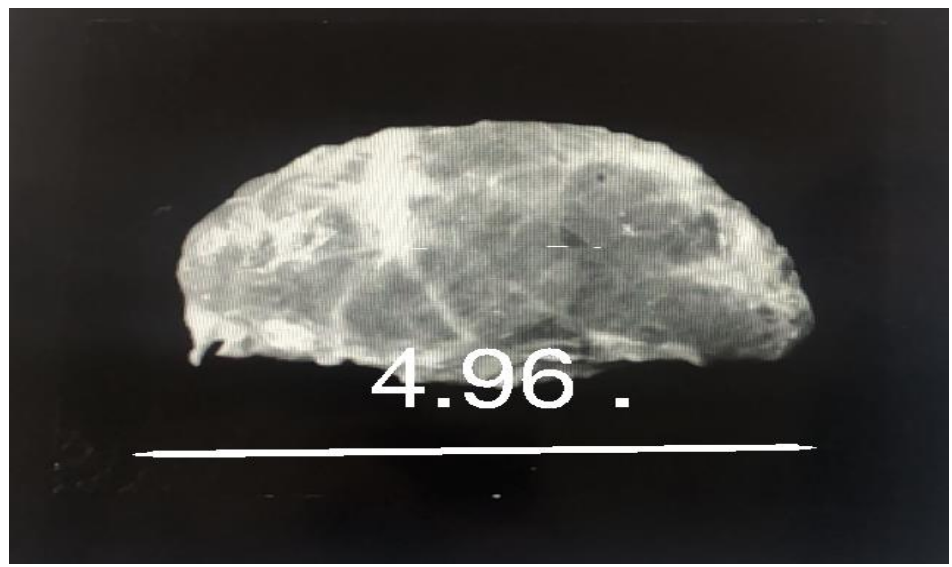

Fig. 1. The first variant of the thyroid gland form in the maral (length $4.96 \mathrm{~cm}$ ).

The second option - the left lobe has an elongated shape, narrows downwards, passing into a small process, the inner surface is concave. In the right lobe, the process has a shorter length. The weight of the left lobe of the gland is $9.8 \pm 1.2 \mathrm{~g}$, the weight of the right lobe is $8.6 \pm 0.7 \mathrm{~g}$. Vertical size of the left lobe, including the process $-15.70 \pm 23.0 \mathrm{~cm}$, transverse $2.10 \pm 0.20 \mathrm{~cm}$, thickness $-1.3 \mathrm{~cm}$. Vertical size of the right lobe with the process is $7.10 \pm 0.20 \mathrm{~cm}$, transverse $-2.30 \pm 0.53 \mathrm{~cm}$, thickness $-1.2 \mathrm{~cm}$.

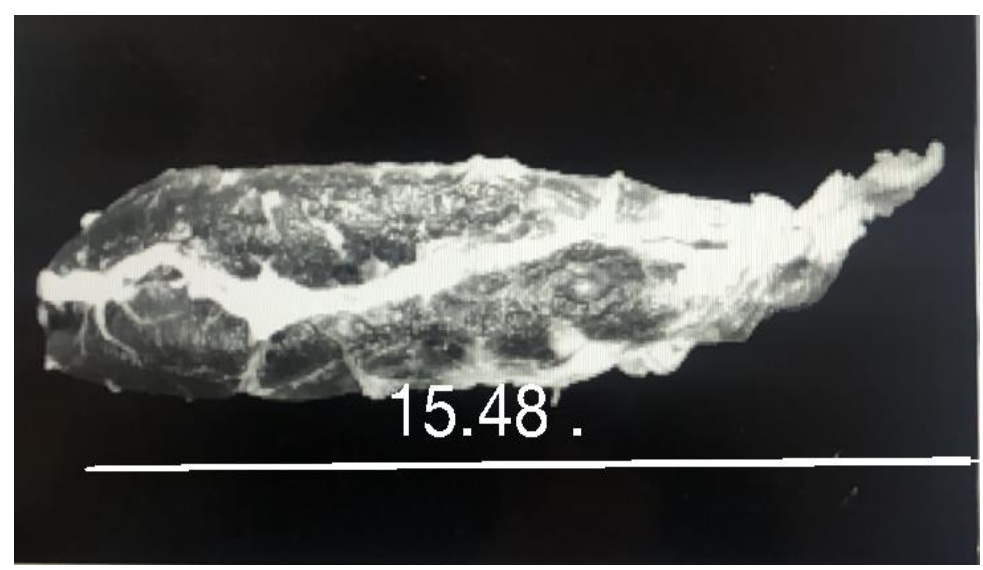

Fig. 2. The second option of the thyroid gland shape (length $15.48 \mathrm{~cm}$ ). 
In the dapple deer, the weight of one gland lobe is $4.0 \pm 0.2 \mathrm{~g}$. The gland itself is an elongated oval, its lobes are flattened, have a red-brown color, their vertical size is $4.50 \pm 0.20 \mathrm{~cm}$, transverse $-1.70 \pm 0.10 \mathrm{~cm}$, thickness $-0.50 \mathrm{~cm}$.

In roe deer, the weight of one lobe of the gland is $2.0 \pm 0.2 \mathrm{~g}$. The gland is dark gray in color, of elongated oval shape, has a delicate fine-grained structure. The vertical size of the lobes is $3.80 \pm 0.20 \mathrm{~cm}$, the transverse size is $0.14 \pm 0.03 \mathrm{~cm}$, and the thickness is $0.33 \mathrm{~cm}$.

In moose, the weight of one lobe of the thyroid gland is $14.0 \pm 0.5 \mathrm{~g}$. The organ has a red-brown color, flat horseshoe shape, close to triangular shape, a rigid coarse-grained structure. The vertical size of the lobe is $4.70 \pm 0.30 \mathrm{~cm}$, the transverse size is $2.90 \pm 0.10 \mathrm{~cm}$, and the thickness is $0.7 \mathrm{~cm}$.

Histological examination of the gland revealed that it is covered with two connective tissue capsules. The structure of the Cervidae's own thyroid capsule is very delicate and loose, so it is not completely preserved when making histological sections. The thickness of own capsule in the maral is $14.58 \pm 1.96$ microns, moose - $17.22 \pm 2.89$ microns, dapple deer - $14.60 \pm 1.52$ microns, roe deer $-10.11 \pm 1.75$ microns. The blood supply to the thyroid gland is provided by two lower thyroid and two upper thyroid arteries, and it is also surrounded by a well-defined venous plexus located between the fibrous and fascial capsules.

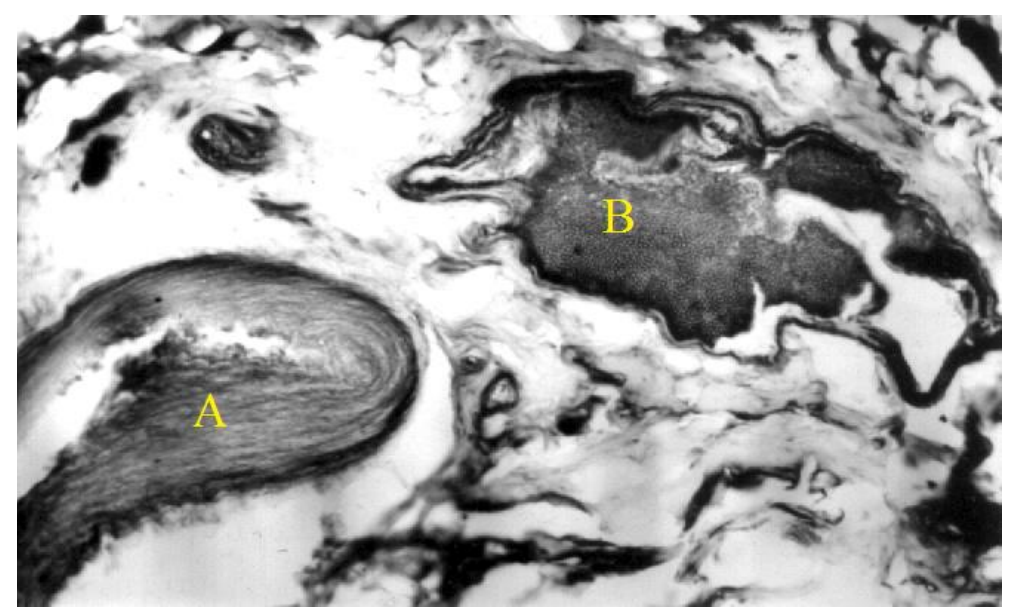

Fig. 3. Artery and vein of the external capsule (A - artery, B - vein). Formalin. Hematoxylin-eosin. (amp. 7x20)

The parenchyma is located under the capsule. The main weight of parenchymal cells is made up of thyrocytes, mainly of a cubic shape, forming the walls of the follicles and lying in a single layer. The nuclei of thyrocytes are rounded, located closer to the basal edge. We have established the heterogeneity of the follicles location in the thyroid gland of maral, dapple deer and moose. In maral and moose, follicles of a larger diameter are located in the center of the organ, and small follicles are located on the periphery. The same structure of the thyroid gland is noted by I.A. Antipin in the European reindeer [1]. 


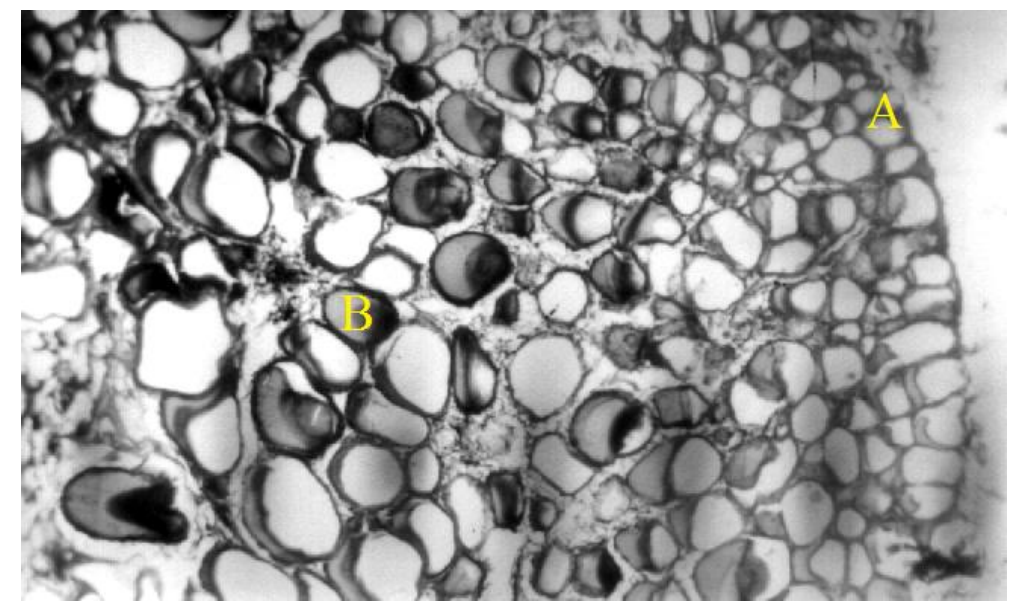

Fig. 4. The follicles location in the thyroid gland of the moose (A - small follicles on the gland periphery, B - large follicles in the center of the gland). Formalin. Hematoxylin-eosin. (amp. 7x8)

In the dapple deer, the thyroid gland does not have a clearly defined heterogeneity: large follicles are located in the center, and of a smaller diameter - both in the center and on the periphery of the gland. In the roe deer, the gland follicles are also not located zonally, they are almost the same size in the center and on the gland periphery. The follicles lumen is evenly filled with a pinkish colloid, often foamy at the edges.

In the morphometric study of the glands of Cervidae family representatives, we obtained the following data (Table 1).

Table 1. Morphometric parameters of the thyroid gland of some ungulates representatives

\begin{tabular}{|l|l|l|l|l|}
\hline Indicators & Moose & Maral & Dapple deer & Roe deer \\
\hline $\begin{array}{l}\text { Height } \\
\text { thyrocytes, } \\
\text { microns }\end{array}$ & $4.47 \pm 0.21$ & $4.78 \pm 0.12^{*}$ & $4.11 \pm 0.21$ & $4.23 \pm 0.22^{*}$ \\
\hline $\begin{array}{l}\text { Volume of } \\
\text { thyrocyte } \\
\text { nuclei, microns }\end{array}$ & $27.33 \pm 3.56^{*}$ & $46.57 \pm 2.80^{*}$ & $28.50 \pm 3.93^{*}$ & $34.74 \pm 6.03$ \\
\hline $\begin{array}{l}\text { Follicles } \\
\text { diameter, } \\
\text { microns }\end{array}$ & $153.58 \pm 7.46^{* *}$ & $171.90 \pm 6.49^{* *}$ & $137.59 \pm 7.32$ & $128.09 \pm 9.45^{* *}$ \\
\hline Brown index & 34.36 & 35.96 & 33.48 & 30.28 \\
\hline
\end{tabular}

Note: the differences between neighboring groups are significant: ${ }^{*}$ - at $\mathrm{P}<0.05$; ${ }^{*}$ - at $\mathrm{P}<0.01$.

The analysis of the obtained data shows that the thyroid gland of the roe deer has the minimal diameter of the follicles, and in maral - the largest. Differences between these indicators are significant in roe deer and maral, roe deer and moose, moose and maral, dapple deer and moose, dapple deer and maral $(\mathrm{P}<0.01)$. The differences between the follicles diameters of roe deer and dapple deer are unreliable.

The height of thyrocytes is minimal in dapple deer, it increases in roe deer and moose, and it is maximal in maral. The differences are significant $(\mathrm{P}<0.05)$ between the height of the thyrocytes of roe deer and maral, roe deer and moose, dapple deer and maral. The differences are unreliable between the height of the thyrocytes of maral and moose, roe deer and dapple deer, moose and dapple deer.

The volume of thyrocyte nuclei is minimal in moose, increases in dapple deer, roe deer, and is maximal in maral. The differences between the nuclei volumes are significant $(\mathrm{P}<0.05)$ in moose and maral, maral and dapple deer, maral and roe deer. The differences 
are unreliable between the volumes of thyrocyte nuclei in moose and dapple deer, moose and roe deer, roe deer and dapple deer.

When assessing the state of the colloid in the above-mentioned animals, it can be concluded that the colloid has a homogeneous consistency, is compacted and is intensively stained with hematoxyl-eosin, resorption vesicles are in small quantities. This histological picture indicates a reduced functional state of the thyroid gland of ungulates in winter, which is consistent with the data of other authors $[1,2,5,8]$.

Conclusions. Thus, analyzing the above, we can say that we have established the characteristic morphological features of the thyroid gland in maral, roe deer, moose, and dapple deer. The differences relate not only to the size, shape of the lobes and width of the connective tissue capsule, the histological structure of the above types is different. Maral and moose have a similar gland structure (zoning in the arrangement of follicles of different diameters), in dapple deer, the follicles of smaller diameter are arranged in islands, and in roe deer, the gland follicles have a uniform structure. The nature of heterogeneity does not reflect the systematic affiliation of the ungulate animal. Maral and dapple deer belong to the same genus, but the structure of the thyroid gland is similar in structure in maral and moose. In addition, such morphological parameters of the thyroid gland as the diameter of the follicles, the height of the thyrocytes are also different in representatives of the Cervidae family living in the Altai. The diameter of the follicles is not related to the gland weight (the maral has the largest diameter of the follicles)

\section{References}

1. I.A. Antipin, Morphofunctional features of the thyroid gland structure in reindeer of the Bolshezemelskaya tundra, Biochemical and biophysical mechanisms of physiological functions: Mater. conf. of young physiologists and biochemists. $\mathrm{S}$. $\mathrm{Pb}$, 43-45 (1995).

2. I.A. Antipin, Yu.V. Antipina, Age-related and seasonal changes in the level of thyroid hormones in the blood of reindeer of the Bolshezemelskaya tundra: Mater. of the 13 Komi Republic young. scientific conference, Syktyvkar, 86 (1997)

3. V.A. Zyryanov, Variability of morphophysiological parameters of wild reindeer of the Taimyr population, Ecology, protection and economic use of wild reindeer: a collection of scientific papers, 167 (Novosibirsk, Siberian branch of VASKHNIL, 1985)

4. V.G. Lunitsyn, Antler reindeer husbandry of Russia, 248-257 (RASKHN, Siberian department of the VNIIPO Barnaul, 2004)

5. N.D. Ovcharenko, O.E. Vlasova, O.G. Gribanova, Russian Journal of Developmental Biology, 47 (6), 320-325 (2016)

6. E. Skovorodin, R. Mustafin, S. Bogoliuk, G. Bazekin, V. Gimranov, Veterinary World, 13 (4), 774-781 (2020)

7. Savitha Veerahanumaiah, K.R. Dakshayani, Sharada B. Menasinkai, International Journal of Research in Medical Sciences, 3 (1) (2015)

8. Zdeněk Peksa, Jan Trávníček, Hana Dušová, Roman Konečný and Lucie Hasoňová, Journal of Agrobiology, 28 (1), 79-84, (2011)

9. F. Jelínek, I. Krabačová, V. Kroupová, Acta Vet Brno, 72, 11-16 (2003) 
10. X. Teng, Z. Shan, W Teng, C. Fan, H. Wang, R. Guo, Clin Exp Med, 9, 51- 59 (2009) 